\title{
Melioidosis - A Rare Cause of Neck Abscess in Immunocompromised
}

https://doi.org/10.47210/bjohns.2021.v29i1.405

Nayana V G, ${ }^{1}$ Sai Manohar $S,{ }^{1}$ Nandini Raveendra, ${ }^{1}$ Sheetal Rai, ${ }^{1}$ Preethishree $P^{2}$

Introduction

\section{ABSTRACT}

Melioidosis is an infectious disease caused by Burkholderia pseudomallei, a saprophytic bacterium found in soil and water. Though multiple abscesses are a common presentation of melioidosis, isolated neck abscess due to B. pseudomallei is extremely rare and it is more prevalent in immunocompromised people.

\section{Case Report}

A young woman with uncontrolled Type II diabetes presented with neck abscess. Abscess was drained and culture showed Burkholderia pseudomallei. Appropriate treatment was given, including 3 weeks of parenteral antibiotics and 3 months of oral prophylactic antibiotics to prevent relapses. Patient responded well to treatment.

$\underline{\text { Discussion }}$

Increased awareness of opportunistic infections like melioidosis is essential in the present era as diabetes is emerging as a global pandemic. It clinically resembles tubercular neck abscess. Strong clinical suspicion, early intervention and long term antibiotics can cure the disease completely without relapse.

$\underline{\text { Kevwords }}$

Melioidosis; Abscess; Immunocompromised Host; Burkholderia pseudomallei

$\mathrm{M}$ elioidosis, a multisystem disease is caused by a gram negative saprophytic bacteria, Burkholderia pseudomallei. It has a wide range of clinical presentation ranging from a localized abscess to severe multisystem infection. This environmental bacteria seen in the wet soil of the tropical region, especially in paddy fields. ${ }^{1}$ Melioidosis is an endemic disease in the geographical belt of southeast Asia and northern Australia, but recently many cases have been reported in South India, China, America and Taiwan. ${ }^{2}$ Melioidosis occurs both in humans and animals. Melioidosis is an opportunistic infection seen in immunocompromised individuals and diabetes mellitus is a single strong risk factor for the same. ${ }^{3}$ Systemic disease is associated with very high mortality.

1 - Department of ENT, Yenepoya Medical College

2 - Department of Microbiology, Yenepoya Medical College

\section{Corresponding author:}

Dr Nayana V G

email: drnayanavg@yenepoya.edu.in
Melioidosis presenting as an isolated neck abscess is scarcely reported in English literature. Early diagnosis is very important as antibiotic regimen and duration of treatment is different from other common neck abscesses. Patient requires prolonged treatment failure of which may lead to relapse of the disease

\section{Case Report}

A33 year old woman, hailing from the coastal belt of south east Asia, presented with a painful swelling on the left side of the neck. She was diagnosed with type II Diabetes Mellitus 6 years back and was on oral hypoglycemic agents. The case is being reported after obtaining informed consent from the patient for publication. She was a dairy farmer and was rearing cattle at home. Her symptoms started two weeks prior to presentation, as a small swelling which gradually progressed and was associated with fever. She had received parenteral broad spectrum antibiotics (Piperacillin + Tazobactam and Metronidazole) from another hospital however her 


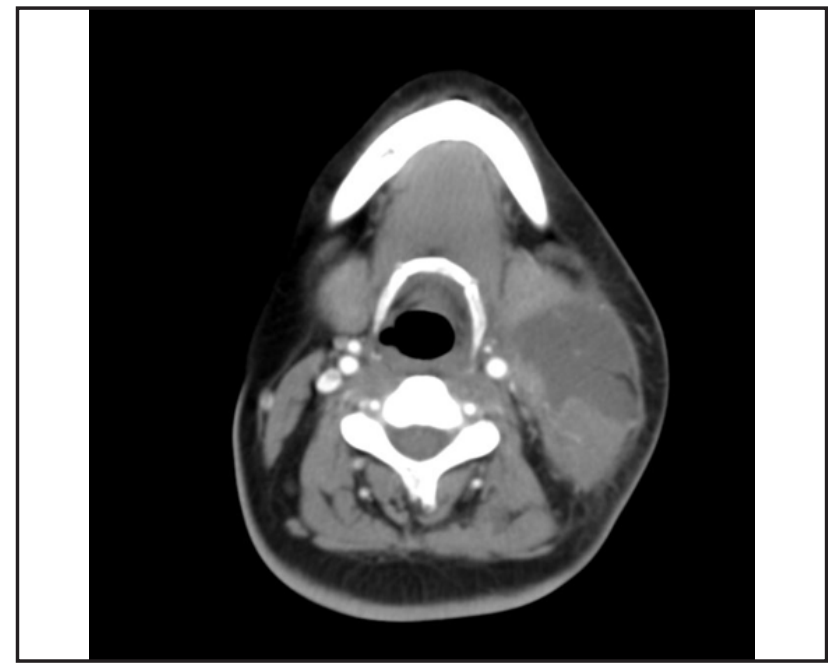

Fig. 1. Contrast enhanced CT scan Neck Axial section collection in left side of neck with central necrosis abutting parotid gland

symptoms did not improve. She did not give history of dental caries, tuberculosis or recurrent infection in head and neck region. On examination, there was a smooth swelling of $10 \times 8 \mathrm{~cm}$ on the left side of the neck with local rise of temperature and tenderness. She had grade 2 trismus.

Haematological investigations were done and she was found to have very poor control of blood sugar

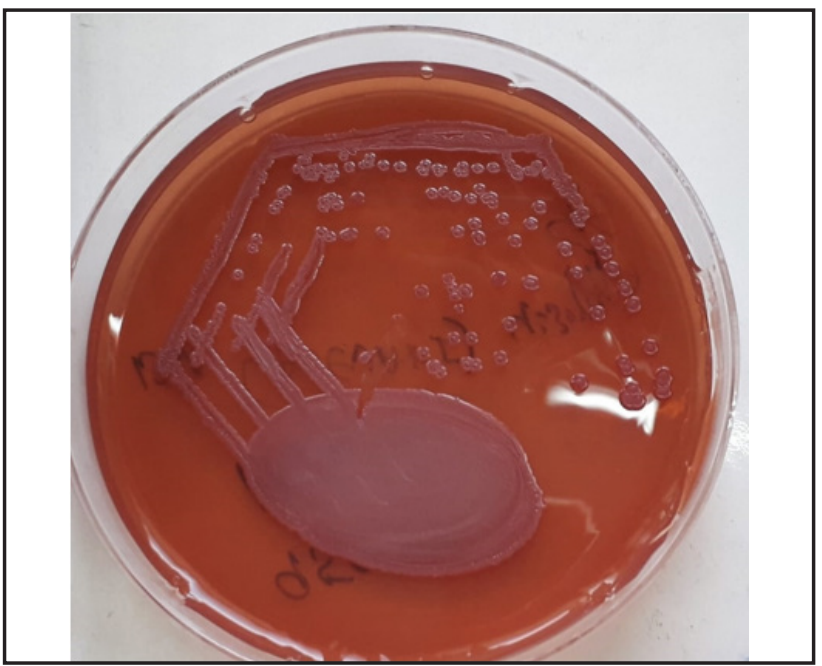

Fig. 3. MacConkey agar showing dry wrinkled colonies with metallic sheen

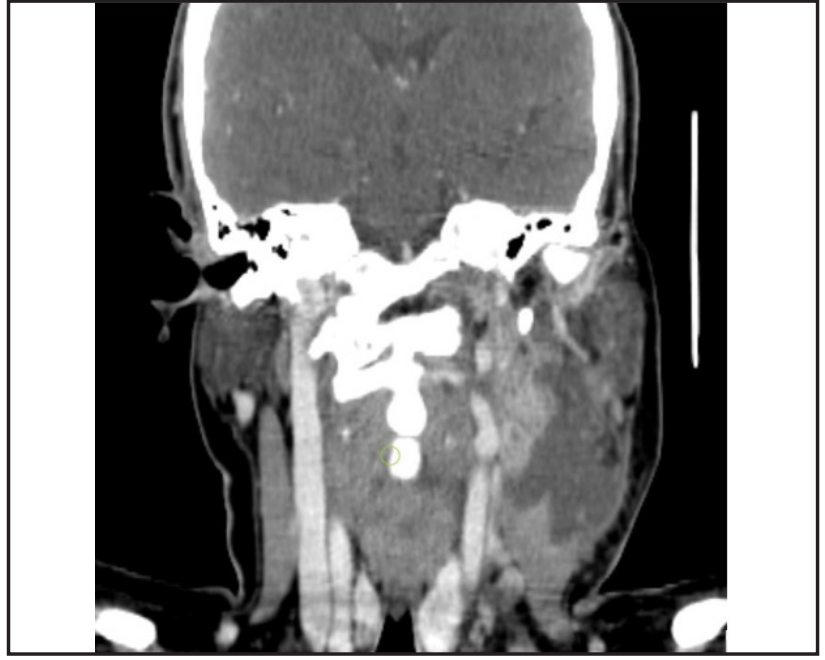

Fig. 2. Contrast enhanced CT scan Neck coronal section collection in left side of neck with central necrosis abutting carotid artery

$($ Glycosylated hemoglobin $=12.1 \%)$. She was started on insulin for better control of blood sugar. Rest of the investigations were normal. Ultrasonogram of the neck revealed a thick collection on the left side of the neck closely abutting the parotid gland. $10 \mathrm{ml}$ pus was aspirated with ultrasound guidance and sent for culture and antibiotic sensitivity. Swelling reduced in size and trismus improved following aspiration. Contrast

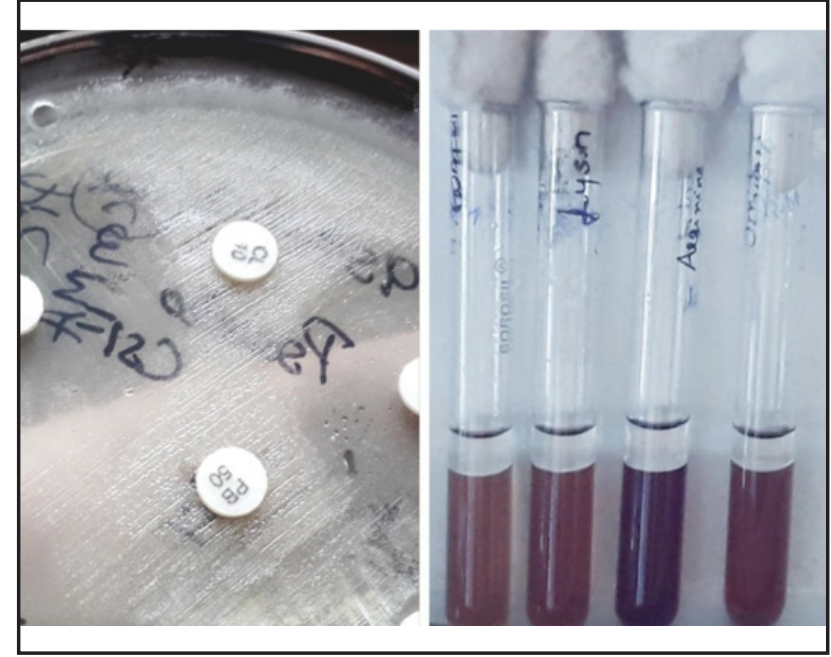

Fig. 4. Bacterial colonies showing Polymyxin B and Colistin resistance 


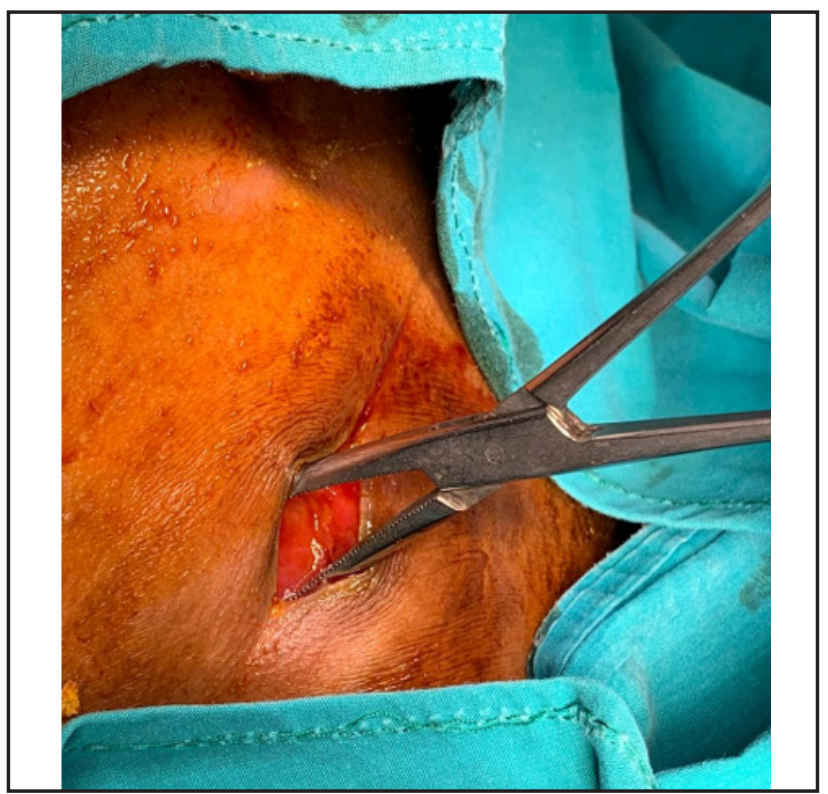

Fig. 5. Clean wound after 2 weeks of incision and drainage

enhanced computed tomography (Iohexol $1 \mathrm{mg} / \mathrm{kg}$ ) of neck showed an ill defined necrotic peripherally enhancing collection in the left side of neck involving sternocleidomastoid muscle and closely abutting carotid sheath and internal jugular vein. (Figs. 1,2)

Abscess was managed by incision and drainage under general anesthesia. Skin crease incision was made and all loculated collections were opened up without injuring the carotid sheath. Thick pus of approximately $10 \mathrm{ml}$ was drained and sent for culture and sensitivity. Pus culture showed growth of Burkholderia pseudomallei,(Figs. $3,4)$ sensitive to Ceftazidime and Imipenem. GeneXpert for tuberculosis was negative.

Full evaluation for systemic manifestations of melioidosis was done, including blood culture and sonogram of visceral organs, which was normal. She was treated with Intravenous Ceftazidime $2 \mathrm{gm}$ every 8 hourly for 3 weeks. Daily dressing of the wound cavity was done. Swab was taken from wound cavity 2 weeks later, which was sterile. Wound was clean (Fig. 5), and secondary suturing was done. Oral Cotrimoxazole twice daily for 3 months was given as maintenance therapy. She was followed up for 1year. Her symptoms had resolved completely with no clinical evidence of relapse

\section{Discussions}

Burkholderia pseudomallei was discovered by Major Alfred Whitmore and CS Krishnasami in Rangoon, which is present day Burma, in 1912, hence Melioidosis is also known as Whitmore's disease/Rangoon's disease.1 B. pseudomallei belongs to Burkoholderia genus which contains upto 40 species. B. mallei which is responsible for Glanders disease in horses and highly virulent diseases in humans also belongs to the same family. ${ }^{4}$

B. pseudomallei grows in humid environment and wet soil and hence is commonly seen among farmers. Incidence of infection peaks during rainy seasons. It is more commonly seen in adults but also reported in children. ${ }^{5}$ The patient in this case study was a dairy farmer and she gave history of recent unnatural cattle death at home. Incubation period of disease varies from 1 day to 3 weeks. Sometimes bacteria may lie dormant in host for many years and manifest disease when immunity is compromised. ${ }^{3}$ The modes of transmission of infection are percutaneous inoculation through skin injuries, inhalation of aerosolised bacteria and even ingestion of mastitis-affected breast milk. ${ }^{6}$

Clinical features depend upon the mode of inoculation, strain of bacteria and risk factors of the host. Melioidosis is an opportunistic infection and hence is more frequently seen in patients with immunosuppressive illness like diabetes mellitus, chronic pulmonary disease, chronic renal failure, or cancer. ${ }^{7}$ A very strong association between diabetes and melioidosis is observed. This patient in this case report was diabetic with poor blood glucose control which probably was the predisposing factor.

Melioidosis is referred as a 'remarkable imitator'. Clinical manifestations may vary from acute fulminant disease or a chronic debilitating localized infection. It may present as pneumonia, musculoskeletal infections, abscess (skin, dental and visceral) or acute fulminant septicemia. Pneumonia is the most common presentation. ${ }^{8}$ Head and neck abscesses are very rarely observed. Systemic disease is associated with very high mortality 
Melioidosis is diagnosed by growing the bacteria in culture media from the clinical sample. Since it is an uncommon bacterium with a very high chance of misidentification in laboratory, a proper communication between clinician and microbiologist is needed in suspicious cases. Another test is Polymerase Chain Reaction test of serum, which is not useful in endemic areas where there are more than $50 \%$ asymptomatic seropositive cases. ${ }^{9}$

Treatment regimen of melioidosis is quite different from other commonly seen neck abscesses. It consists of 2 weeks of intensive phase and 3-6 months of eradication phase of antibiotics. Antibiotics are given for longer duration due to protracted course of the disease and to prevent relapse. Neck abscess is managed by surgical drainage along with antibiotics. Intravenous Ceftazidime, Meropenem or Imipenem are given during intensive phase and Co-trimoxazole is recommended for eradication phase. Garas et al has reported a case of discharging meliodosis neck abscess treated with vacuum assisted closure device which helped in quick healing of abscess. ${ }^{10}$ Amoxicillin-Clavulanic acid is advised for children and pregnant woman, for whom cotrimoxazole is contraindicated nevertheless, it is associated with a high relapse rate. ${ }^{11}$

Compliance to eradication phase is very important to prevent the relapse of disease. Increased awareness and clinical suspicion of opportunistic infections like melioidosis is paramount as diabetes mellitus is becoming a global pandemic. Early diagnosis of melioidosis is very important as antibiotic regimen and duration of treatment is different from other pyogenic neck abscess. Patient requires prolonged treatment, failure of which may lead to relapse of the disease

\section{References}

1. White NJ. Melioidosis. Lancet 2003; 361:1715-22

2. Dance DA. Melioidosis as an emerging global problem. Acta Trop. 2000; 74:115-9

3. Currie BJ. Advances and remaining uncertainties in the epidemiology of Burkholderia pseudomallei and melioidosis. Trans R Soc Trop Med Hyg. 2008;102:225-7.

4. Stewart T, Engelthaler DM, Blaney DD, Tuanyok A, et al. Epidemiology and investigation of melioidosis, Southern Arizona. Emerg Infect Dis. 2011; 17:1286-8

5. Pagnarith Y, Kumar V, Thaipadungpanit J, Wuthiekanun V, et al. Emergence of pediatric melioidosis in Siem Reap, Cambodia. Am J Trop Med Hyg. 2010; 82:1106-12

6. Ralph A, McBride J, Currie BJ. Transmission of Burkholderia pseudomallei via breast milk in Northern Australia. Pediatr Infect Dis J. 2004; 23:1169-71

7. Raja NS. Localized melioidosis. J Pak Med Assoc. 2003; $53: 373-4$

8. Currie BJ, Ward L, Cheng AC. The epidemiology and clinical spectrum of melioidosis: 540 cases from the 20 year Darwin prospective study. PLoS Negl Trop Dis. 2010; 4:e900

9. Chantratita N, Wuthiekanun V, Limmathurotsakul D, Thanwisai A, et al. Prospective clinical evaluation of the accuracy of $16 \mathrm{~S}$ rRNA real-time PCR assay for the diagnosis of melioidosis. Am J Trop Med Hyg. 2007; 77:814-7

10. Garas G, Ifeacho S, Millard R, Tolley N. Melioidosis and the vacuum-assisted closure device: a rare cause of a discharging neck wound, and a new approach to management. J Laryngol Otol. 2010;124(9):1021-4

11. Peacock SJ, Schweizer HP, Dance DA, Smith TL, et al Management of accidental laboratory exposure to Burkhold

12. -eria pseudomallei and B. mallei. Emerg Infect Dis. 2008; 14:e2. 